\title{
Tuberculosis intestinal, una entidad poco frecuente de abdomen agudo
}

\author{
Intestinal tuberculosis, a rare entity of acute abdomen
}

\author{
Jorge Andrés Myers Esmenjaud, * José Martín Hernández Márquez, \\ Héctor Leonardo Pimentel Mestre, ${ }^{\ddagger}$ Analy Mendoza Gómez, ${ }^{\S}$ Patricia Aidé López Olmos, \\ Moisés Olvera Vidal, "Estela Alejandra Brito González"
}

Citar como: Myers EJA, Hernández MJM, Pimentel MHL, Mendoza GA, López OPA, Olvera VM et al. Tuberculosis intestinal, una entidad poco frecuente de abdomen agudo. Acta Med GA. 2022; 20 (1): 67-70. https://dx.doi.org/10.35366/103559

\section{Resumen}

La tuberculosis es una enfermedad infecciosa endémica en México, la cual es causada por Mycobacterium tuberculosis, un bacilo ácido-alcohol resistente. La principal afección orgánica es a nivel pulmonar; sin embargo, se ha reportado que hasta $25 \%$ de los casos pueden tener una afección extrapulmonar, ya sea por diseminación linfática o hematógena, y en casos aislados como primoinfección extrapulmonar. Presentamos el caso de un masculino de 62 años de edad con un cuadro de dolor abdominal de siete días de evolución asociado con melena, en la que se documentaron múltiples perforaciones ileales en el borde antimesentérico con diagnóstico histológico de tuberculosis intestinal. Esta entidad debe sospecharse en pacientes con tratamiento inmunosupresor crónico y deben realizarse medidas de escrutinio de manera preventiva.

Palabras clave: Tuberculosis intestinal, perforación intestinal, abdomen agudo, tuberculosis extrapulmonar.

\section{INTRODUCCIÓN}

La Organización Mundial de la Salud (OMS) en 2020 reportó cerca de 10 millones de personas contagiadas de tuberculosis en todo el mundo, la mayoría son hombres, seguido de mujeres y en menor proporción niños (5.7 millones, 3.2 millones y 1.1 millones, respectivamente).

\section{Abstract}

Tuberculosis is an endemic infectious disease in Mexico, caused by Mycobacterium tuberculosis, an acid-fast stainpositive bacillus. The most common clinical scenarios are pulmonary affections. However, it has been reported that up to $25 \%$ of the cases may present as an extrapulmonary disease, either due to lymphatic or hematogenous dissemination, and isolated cases have been reported as an extrapulmonary primary infection. We present the case of a 62-year-old male with a 7-day history of abdominal pain associated with melena, in which multiple ileal perforations were documented in the anti-mesenteric border with a histological diagnosis of intestinal tuberculosis. This entity should be suspected in patients with chronic immunosuppressive treatment and special screening should be performed to prevent these clinical scenarios.

Keywords: Intestinal tuberculosis, intestinal perforation, acute abdomen, extrapulmonary tuberculosis.
* Médico Residente de la Especialidad en Cirugía General del Hospital Angeles Pedregal. Facultad Mexicana de Medicina de la Universidad La Salle. México.

‡ Médico Especialista en Cirugía General del Hospital General de Zona No. 47 del Instituto Mexicano del Seguro Social. México.

$\S$ Médico Especialista en Anatomía Patológica del Hospital General de Zona No. 47 del Instituto Mexicano del Seguro Social. México.

^ Médico Residente de la Especialidad en Medicina Interna del Hospital Angeles Pedregal. México.
"I Médico Interno de Pregrado del Hospital Angeles Pedregal. México.

Correspondencia:

Jorge Andrés Myers Esmenjaud

Correo electrónico: amyers_med@hotmail.com

Aceptado: 11-02-2021.

www.medigraphic.com/actamedica
De tal manera que para ese año se registraron 1.5 millones de defunciones atribuibles a esta enfermedad, con una tasa de mortalidad calculada de $42 \%$ según la OMS. Por su parte, el Instituto Nacional de Salud Pública de México indicó que durante el año 2018 más de 1,800 mexicanos murieron por tuberculosis o por secuelas de la misma, destacando que $90 \%$ fueron muertes directas por 
tuberculosis pulmonar. ${ }^{1-3} \mathrm{~A}$ finales de febrero de 2019 ya se tenía registro de al menos 792 casos nuevos de tuberculosis, de los cuales 667 fueron mujeres y 125 hombres, esto de acuerdo con cifras del Boletín Epidemiológico de la Secretaría de Salud. ${ }^{4,5}$

El principal órgano afectado es el pulmón, no obstante, existen casos con presentación en otros órganos, ya sea por diseminación hematógena, linfática o como sitios primarios. Uno de los sitios extrapulmonares afectados es el abdomen, en el que se puede presentar en cuatro formas: linfadenopatía tuberculosa, tuberculosis peritoneal, tuberculosis gastrointestinal y tuberculosis visceral que afecta a los órganos sólidos. El diagnóstico de abdomen agudo por tuberculosis puede ser difícil, ya que se presenta con características clínicas y radiológicas inespecíficas, además de que requiere un alto grado de sospecha por parte del cirujano.

\section{CASO CLÍNICO}

Se presenta el caso de un masculino de 62 años de edad, originario y residente de la Ciudad de México, con antecedente de hipotiroidismo y artropatía no especificada en tratamiento con esteroides y antiinflamatorios no esteroideos desde hace cinco años. Se presentó al servicio de urgencias con dolor abdominal de una semana de evolución, que inició de manera inespecífica en epigastrio y que posteriormente se volvió generalizado, opresivo y gradualmente progresivo en intensidad hasta volverse incapacitante. Negó algún factor desencadenante específico, refirió episodios de melena en días previos. Como parte del abordaje se realizaron estudios de laboratorio, se documentó la presencia de

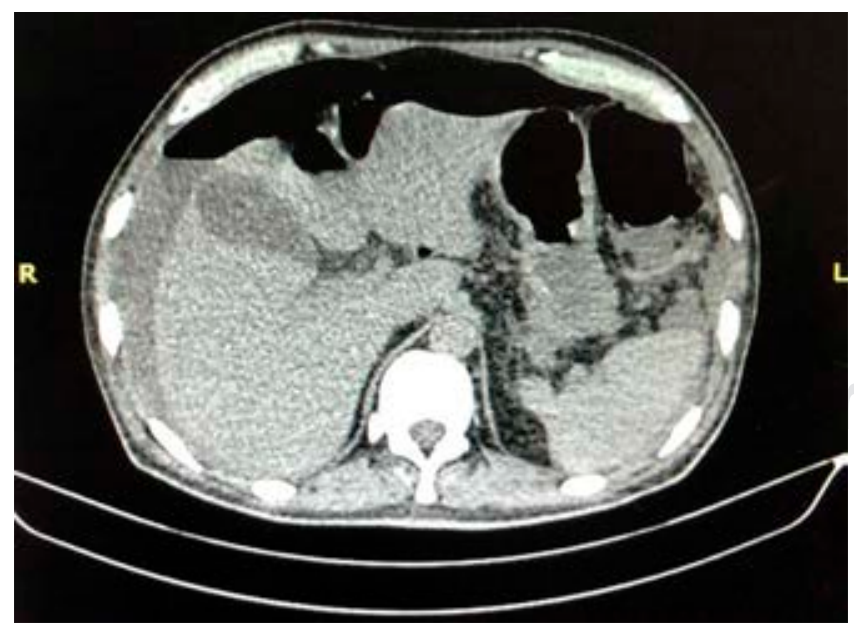

Figura 1: Tomografía de abdomen con evidencia de aire y líquido libre en cavidad. leucocitosis a expensas de monocitos, y de gabinete con una tomografía abdominal en la que se observó aire y líquido libre en cavidad abdominopélvica (Figura 1). Clínicamente con datos de irritación peritoneal y choque séptico de origen abdominal.

Se realizó manejo para estabilización hemodinámica, posteriormente se realizó laparotomía exploradora de urgencia en la que se encontró peritonitis generalizada y múltiples perforaciones rodeadas de parches necróticos a 90, 110, 170, 210 y $300 \mathrm{~cm}$ de la válvula ileocecal, todas en el borde antimesentérico (Figuras 2 y 3). Durante este procedimiento, y a pesar de las medidas realizadas para lograr un control hemodinámico adecuado, el paciente presentó paro cardiorrespiratorio durante el procedimiento quirúrgico.

Se procedió a la resección de un segmento perforado de íleon para su estudio histopatológico, donde posteriormente en la tinción de hematoxilina y eosina se observaron granulomas no caseificantes con inflamación crónica moderada y células gigantes tipo cuerpo extraño. Se realiza tinción de Ziehl-Neelsen revelando bacilos ácido-alcohol resistentes compatibles con tuberculosis (TB) intestinal (Figura 4).

\section{DISCUSIÓN}

La tuberculosis abdominal es una entidad que tiene la característica de mostrar manifestaciones clínicas en relación con la cronicidad de la infección y así mismo es un indicativo de su diseminación. Como patología única o concomitante involucra un reto en el diagnóstico, ya que puede extenderse hacia áreas como el peritoneo, ganglios linfáticos, órganos viscerales sólidos y hacia el tracto intestinal; he aquí la importancia, ya que esta última se puede presentar en cualquier sitio, con predilección hacia la región ileocecal. ${ }^{2}$ Es así como la variabilidad en su presentación y su similitud lleva al manejo quirúrgico a ser una herramienta diagnóstica y un posible tratamiento coadyuvante. ${ }^{3}$

De esta manera, dentro de los estudios epidemiológicos realizados por Estados Unidos, se muestra que de aquellos pacientes que tienen tuberculosis, $18.7 \%$ presentan una manifestación extrapulmonar y dentro de ellas $4.7 \%$ mostraron peritonitis tuberculosa. ${ }^{4}$

Su diagnóstico se basa en la sospecha como proceso de exclusión; sin embargo, se debe hacer mediante el estudio microbiológico, como lo es la tinción de Ziehl-Neelsen que demuestra la presencia de bacilos ácido-alcohol resistentes. ${ }^{5,6}$

Esto en total debe ser tomado en cuenta debido a que en mayor frecuencia la tuberculosis afecta a poblaciones vulnerables como países en desarrollo; dentro de éstos, México, donde existen diversos factores de inmunocom- 

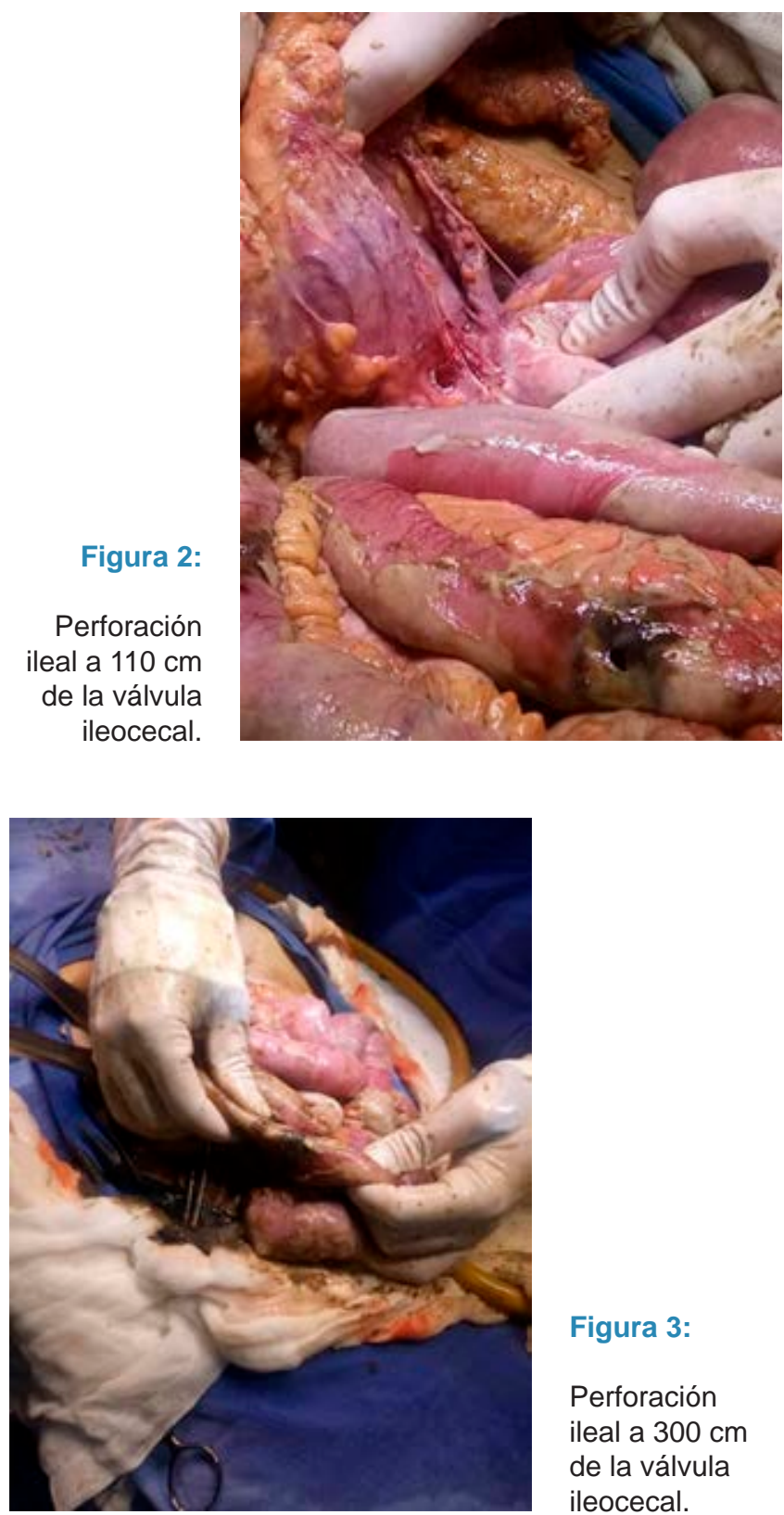

Figura 3:

Perforación ileal a $300 \mathrm{~cm}$ de la válvula ileocecal.

promiso como enfermedades crónico-degenerativas en contraste con países europeos donde su incidencia anual es sólo de 10 casos por cada 100,000. ${ }^{6}$

En la actualidad, la tuberculosis abdominal adquiere una probabilidad diagnóstica en ascenso y una mejora en la resolución de las complicaciones; sin embargo, en el análisis quirúrgico de esta patología, se evidencia una serie de intervenciones recurrentes debido a cuadros de obstrucción y perforación asociadas, lo cual representa una parte importante en las emergencias quirúrgicas en sitios endémicos. ${ }^{6,7}$
Es así que la manifestación abdominal como manifestación extrapulmonar es de carácter infrecuente, representa cerca del $10 \%$ de sus presentaciones, integrando como característica su diseminación hematógena y, en consecuencia, la reactivación por focos latentes, lo que aumenta su aparición por cualquier causa de inmunocompromiso, además de la resistencia a fármacos antituberculosos. ${ }^{6,7}$

Una vez que ocurre la reactivación, los pacientes pueden cursar con adherencias fibrosas, distensión abdominal, fiebre, pérdida de peso, diarrea, dolor y malestar abdominal inespecífico; sin embargo, en una menor parte se presentan con ascitis, líquido libre y perforación intestinal, siendo esto una variante simuladora de diferentes procesos patológicos abdominales. ${ }^{7,8}$

Es así que al tomar en cuenta las características de presentación, de acuerdo con sitios endémicos, se posiciona al dolor abdominal como el principal síntoma, seguido de la obstrucción intestinal, hemorragia digestiva baja, perforación intestinal, fiebre, pérdida de peso, historia concomitante de tuberculosis pulmonar, historia de respuesta parcial o nula a antituberculosos en alguna otra presentación tuberculosa. ${ }^{8}$

En el curso del tratamiento quirúrgico de la perforación y la obstrucción intestinal, se muestra que la laparotomía exploradora representa el principal abordaje dentro de la técnica quirúrgica. En relación dentro del seguimiento quirúrgico, las complicaciones postoperatorias representan un cuadro altamente recurrente, involucrando la dehiscencia de la incisión, el desarrollo de un índice de masa corporal

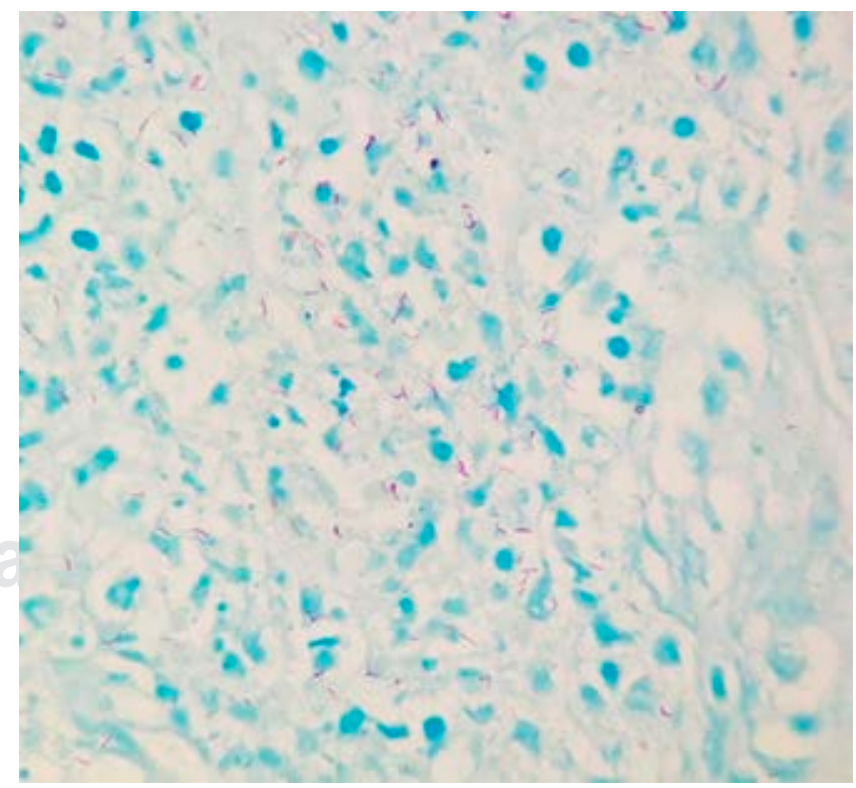

Figura 4: Tinción de Ziehl-Neelsen que revela bacilos ácido-alcohol resistentes compatibles con tuberculosis (TB) intestinal. 
bajo, desarrollo de estomas y colecciones intraabdominales y la muerte secundaria a una diseminación a múltiples órganos y sepsis. ${ }^{9,10}$

De esta manera, una vez confirmado el diagnóstico de tuberculosis abdominal, su asociación hacia la obstrucción y perforación intestinal representa el tema de esta discusión, ya que en su análisis se ve mayor relación hacia los pacientes con un tratamiento previo para tuberculosis de algún otro sitio, en su mayoría pulmonar; si bien como se menciona el seguimiento es de vital importancia, ya que esta patología representa un gran reto en su tratamiento y un gran reto en su resolución; sin embargo, una vez alcanzado el punto de tratamiento, el buen control y la correcta instauración del tratamiento farmacológico la resolución completa puede ser alcanzada y dar como resultado la disminución de las complicaciones de la enfermedad.

\section{CONCLUSIONES}

La tuberculosis intestinal representa un reto diagnóstico debido a la inespecificidad del cuadro clínico, que en su mayoría se realiza de manera tardía. El 10\% se presenta como un abdomen agudo que requiere manejo quirúrgico de urgencia, he aquí la relevancia de que, a pesar de ser una etiología poco frecuente de abdomen agudo, se deba tener en consideración no sólo por el cirujano, sino por todo el personal de salud.

\section{REFERENCIAS}

1. Centro Nacional de Programas Preventivos y Control de Enfermedades (CENAPRECE). Secretaría de Salud. [Consultado 23 de abril del 2020] Disponible en: http://www.cenaprece.salud.gob.mx/programas/ interior/micobacteriosis/tuberculosis/cifras_oficiales.html

2. World Health Organization. Global tuberculosis report 2019 [Internet]. Who.int. 2019 [Access January 1, 2021]. Available in: https://www.who.int/tb/publications/factsheet_global.pdf?ua =1

3. Tuberculosis: la enfermedad infecciosa más mortífera en el mundo [Internet]. Instituto Nacional de Salud Pública. 2021 [Consultado 1 de enero de 2021]. Disponible en: https://www.insp.mx/avisos/5302tuberculosis-enfermedad-infecciosa-mortifera.html

4. Secretaría de Salud. Tuberculosis. Información General de Micobacteriosis [Internet]. gob.mx. 2021 [Consultado 1 de enero de 2021]. Disponible en: https://www.gob.mx/salud/acciones-yprogramas/tuberculosis

5. Romero L. La tuberculosis aún es causa de muerte. Gaceta UNAM. 2021; 5171: 1-3. [Consultado 1 de enero de 2021]. Disponible en: https://www.gaceta.unam.mx/la-tuberculosis-aun-es-causa-de-muerte/

6. Choi EH, Coyle WJ. Gastrointestinal tuberculosis. Microbiol Spectr. 2016; 4 (6). doi: 10.1128/microbiolspec.TNMI7-0014-2016.

7. Aggarwal P, Kedia S, Sharma R, Bopanna S, Madhusudhan KS, Yadav DP et al. Tubercular intestinal strictures show a poor response to anti-tuberculous therapy. Dig Dis Sci. 2017; 62 (10): 2847-2856.

8. Van Slambrouck J, Vlasselaers J, Devos B. A case report of peritoneal tuberculosis diagnosed by laparoscopy in a low prevalence setting. Acta Chir Belg. 2021; 121 (4): 269-273.

9. Singh H, Krishnamurthy G, Rajendran J, Sharma V, Mandavdhare H, Kumar $\mathrm{H}$ et al. Surgery for abdominal tuberculosis in the present era: experience from a tertiary-care center. Surg Infect (Larchmt). 2018; 19 (6): 640-645.

10. Debi U, Ravisankar V, Prasad KK, Sinha SK, Sharma AK. Abdominal tuberculosis of the gastrointestinal tract: revisited. World J Gastroenterol. 2014; 20 (40): 14831-14840. 